\title{
Nationwide analysis of hospital-to-hospital transfer in patients with aneurysmal subarachnoid hemorrhage requiring aneurysm repair
}

\author{
Christopher Roark, MD, MS, ${ }^{1}$ David Case, MD, ${ }^{1}$ Mark Gritz, PhD,, Patrick Hosokawa, MS, ${ }^{5}$ \\ David Kumpe, MD, ${ }^{3}$ Joshua Seinfeld, MD, ${ }^{1,3}$ Craig A. Williamson, MD, ${ }^{6}$ and Anne M. Libby, PhD ${ }^{2}$
}

Departments of ${ }^{1}$ Neurosurgery, ${ }^{2}$ Emergency Medicine, and ${ }^{3}$ Radiology; ${ }^{4}$ Division of Health Care Policy and Research; ${ }^{5}$ Adult and Child Center for Health Outcomes Research and Delivery Science (ACCORDS), University of Colorado, Aurora, Colorado; and ${ }^{6}$ Department of Neurosurgery, University of Michigan, Ann Arbor, Michigan

OBJECTIVE Aneurysmal subarachnoid hemorrhage (aSAH) has devastating consequences. The association between higher institutional volumes and improved outcomes for aSAH patients has been studied extensively. However, the literature exploring patterns of transfer in this context is sparse. Expansion of the endovascular workforce has raised concerns about the decentralization of care, reduced institutional volumes, and worsened patient outcomes. In this paper, the authors explored various patient and hospital factors associated with the transfer of aSAH patients by using a nationally representative database.

METHODS The 2013 and 2014 years of the National Inpatient Sample (NIS) were used to define an observational cohort of patients with ruptured brain aneurysms. The initial search identified patients with SAH (ICD-9-CM 430). Those with concomitant codes suggesting trauma or other intracranial vascular abnormalities were excluded. Finally, the patients who had not undergone a subsequent procedure to repair an intracranial aneurysm were excluded. These criteria yielded a cohort of 4373 patients, 1379 of whom had undergone microsurgical clip ligation and 2994 of whom had undergone endovascular repair. The outcome of interest was transfer status, and the NIS data element TRAN_IN was used to define this state. Multiple explanatory variables were identified, including age, sex, primary payer, median household income by zip code, race, hospital size, hospital control, hospital teaching status, and hospital location. These variables were evaluated using descriptive statistics, bivariate correlation analysis, and multivariable logistic regression modeling to determine their relationship with transfer status.

RESULTS Patients with aSAH who were treated in an urban teaching hospital had higher odds of being a transfer (OR $2.15,95 \% \mathrm{Cl} 1.71-2.72)$ than the patients in urban nonteaching hospitals. White patients were more likely to be transfer patients than were any of the other racial groups $(p<0.0001)$. Moreover, patients who lived in the highest-income zip codes were less likely to be transferred than the patients in the lowest income quartile (OR $0.78,95 \% \mathrm{Cl} 0.64-0.95)$. Repair type (clip vs coil) and primary payer were not associated with transfer status.

CONCLUSIONS A relatively high percentage of patients with aSAH are transferred between acute care hospitals. Race and income were associated with transfer status. White patients are more likely to be transferred than other races. Patients from zip codes with the highest income transferred at lower rates than those from the lowest income quartile. Transfer patients were preferentially sent to urban teaching hospitals. The modality of aneurysm treatment was not associated with transfer status.

https://thejns.org/doi/abs/10.3171/2018.4.JNS172269

KEYWORDS subarachnoid hemorrhage; epidemiology; teaching hospital; intracranial aneurysm; microsurgical clip ligation; endovascular repair; vascular disorders

ABBREVIATIONS aSAH = aneurysmal subarachnoid hemorrhage; ICD-9-CM = International Classification of Diseases, Ninth Revision, Clinical Modification; NIS = National Inpatient Sample.

SUBMITTED September 18, 2017. ACCEPTED April 5, 2018.

INCLUDE WHEN CITING Published online October 5, 2018; DOI: 10.3171/2018.4.JNS172269. 
A NEURYSMAL subarachnoid hemorrhage (aSAH) due to a ruptured brain aneurysm is a significant public health problem because the typically young patients have a high risk of death and survivors have a high rate of disability. Unruptured brain aneurysms may be surgically treatable, especially in advance of a catastrophic rupture. Centers with an increased case volume have been associated with positive outcomes. ${ }^{3,4,8,11,18,20,22}$ Because of the geographic dispersion of patients and subspecialists, patient transfers to higher-volume centers further increase case volume and improve outcomes.

For patients with aSAH, the extent of hospital-to-hospital (interfacility) transfers and their association with outcomes is not well understood. Existing studies are generally retrospective reviews of single institutional databases and are unclear regarding the effect of interfacility transfer on patient outcomes. The aSAH patient presents challenges for interhospital transfer given the risk of aneurysm re-rupture and the potential to develop life-threatening hydrocephalus and malignant intracranial hypertension. Adverse events have been seen in up to $34 \%$ of critically ill patients during hospital transfer, ${ }^{19}$ and significant research has been done to improve critical care transport safety. $2,12,19,25$

Despite the potential effect of transfer characteristics on patient outcomes, there is limited evidence on patterns of interhospital transfer in aSAH patients. Existing studies have examined differences in medical treatment based on race or ethnicity ${ }^{6,16,26}$ and socioeconomic status ${ }^{1,9,15,26}$ among patients with ischemic stroke and $\mathrm{SAH}^{23}$ or unruptured aneurysms. ${ }^{7}$ Brinjikji and colleagues found that insured white women from higher socioeconomic strata were the most likely patients to undergo elective cerebral aneurysm repair?

In the current study, we evaluated patterns of interfacility transfer in a nationally representative sample of patients with aSAH unrelated to trauma. Given the specialized nature of microsurgical and endovascular treatment for aSAH, patterns were based on available demographic and socioeconomic data. We hypothesized that aSAH transfers from acute hospitals are more likely to be sent to urban teaching hospitals and less likely to be sent to nonteaching or rural hospitals.

\section{Methods \\ Patient Population}

An observational cohort of patients with ruptured brain aneurysms not associated with trauma was created using the National Inpatient Sample (NIS) for the years 2013-2014. The primary diagnosis code sought was based on the International Classification of Diseases, Ninth Revision, Clinical Modification (ICD-9-CM) for SAH (430). Part of the Healthcare Cost and Utilization Project (HCUP), the NIS is the largest publicly available all-payer inpatient healthcare database in the United States, yielding national estimates of hospital inpatient stays. The NIS includes 7 million unweighted and 35 million weighted hospital visits annually.

The cohort of 14,532 patients had one visit defined by ICD-9-CM 430. We excluded patients with concomitant codes consistent with head trauma (ICD-9-CM 800.0$801.9,803.0-804.9,850.0-854.9,873.0-873.9)$ or arteriovenous malformation or fistula (747.81; ICD-9-CM procedures $39.53,92.30$ ) or discharge to home or self-care after less than 1 day. The final cohort included only patients treated for a cerebral aneurysm after the aSAH. We excluded 10,159/14,532 (70\%) patients with no microsurgical clip ligation (ICD-9-CM 39.51) or endovascular coiling (ICD-9-CM 39.72, 39.75, 39.76, 39.79). We excluded these patients because they represent a heterogeneous group that did not have definitive treatment for an aneurysm and they may have had intratumoral hemorrhage, infectious vasculitis, or a host of other conditions. The final cohort consisted of 4373 patients with aSAH and a repair procedure: 1379 (31.5\% weighted) had undergone microsurgical clip ligation and 2994 (68.5\% weighted) had undergone endovascular embolization.

\section{Outcome Variable}

The primary outcome of interest was transfer status measured with the NIS binary variable TRAN_IN with a value of 1 indicating transfer to one acute care hospital from a different acute care hospital. Patients identified as having a transfer from another type of health facility were not included as a transfer for the purposes of this study.

\section{Explanatory Variables}

Patient variables of interest were as follows: age (categorized in decades), sex (female), race (white, black, Hispanic, other), admission source, in-hospital death, primary payer, median household income by zip code, length of stay, number of chronic conditions, number of procedures coded on original record, number of days from admission to principal procedure, diagnosis-related group (DRG)based severity or mortality indices, total charges, and final disposition. Hospital variables of interest included hospital control (government, private investor-owned, private nonprofit), hospital size (small, medium, large), hospital census division, hospital location and teaching status (rural, urban nonteaching, urban teaching), and hospital region.

\section{Statistical Analysis}

Descriptive statistics summarized cohort subgroups treated with clipping or coiling and bivariate correlations by transfer status. Weights were applied with PROC SURVEYFREQ (SAS Institute Inc.). Model variables were chosen with bivariate associations, clinical significance, and correlation using a mix of Pearson, Spearman, Cramer's V, and Kendall's Tau tests, depending on the nature of each variable. Multivariable weighted logistic regression (PROC SURVEYLOGISTIC, SAS Institute Inc.) was applied for a multivariate model of interfacility transfer, using reverse stepwise selection for parsimonious models, stratified by clipping or coiling, and we report odds ratio, confidence intervals, $p$ values, and Wald chi-square statistics to differentiate very low $\mathrm{p}$ values.

Table 1 stratifies patient demographics (age, sex, race, primary payer, median household income by zip code), DRG-based severity or mortality indices, and hospitallevel data (hospital type, size, census division, teaching 
TABLE 1. Characteristics of 14,532 SAH patients based on transfer status

\begin{tabular}{|c|c|c|c|c|}
\hline \multirow{2}{*}{$\begin{array}{c}\text { Characteristic (value assigned in } \\
\text { analysis) }\end{array}$} & \multirow[b]{2}{*}{ Variable Name } & \multicolumn{2}{|c|}{ Column \% $(95 \% \mathrm{Cl})$} & \multirow{2}{*}{$\begin{array}{l}\text { p Value, } \\
\text { F-Statistic }\end{array}$} \\
\hline & & No Transfer In & Transfer In & \\
\hline No. of patients* & & 9,945 & 4,587 & \\
\hline Weighted no. of patients* & & 49,725 & 22,935 & \\
\hline Mean age in $\mathrm{yrs}^{*}$ & AGE & $61.0(60.6-61.4)$ & $57.2(56.6-57.8)$ & $<0.0001, N A$ \\
\hline Female sex (1) & FEMALE & $57.8 \%(56.9-58.8)$ & $59.8 \%(58.4-61.1)$ & $0.02,5.33$ \\
\hline Race/ethnicity & RACE & & & $<0.0001,22.13$ \\
\hline White (1) & & $63.1 \%(61.6-64.6)$ & $66.4 \%(63.9-68.9)$ & \\
\hline Black (2) & & $15.2 \%(14.3-16.2)$ & $15.2 \%(13.4-17.0)$ & \\
\hline Hispanic (3) & & $12.4 \%(11.4-13.4)$ & $8.7 \%(7.5-9.9)$ & \\
\hline Other (4-6) & & $9.3 \%(8.5-10.1)$ & $9.7 \%(8.2-11.2)$ & \\
\hline Primary payer & PAY1 & & & $<0.0001,64.81$ \\
\hline Medicare (1) & & $44.3 \%(43.2-45.4)$ & $36.3 \%(34.8-37.8)$ & \\
\hline Medicaid (2) & & $3.4 \%(12.5-14.2)$ & $15.2 \%(13.8-16.5)$ & \\
\hline Private, including $\mathrm{HMO}$ (3) & & $31.4 \%(30.3-32.5)$ & $35.7 \%(34.2-37.2)$ & \\
\hline Other $(4-6)$ & & $10.9 \%(10.2-11.7)$ & $12.9 \%(11.5-14.2)$ & \\
\hline Median household income by zip code & ZIPINC_QRTL & & & $<0.0001,27.85$ \\
\hline$\$ 1-\$ 38,999(1)$ & & $27.2 \%(25.9-28.5)$ & $28.1 \%(26.0-30.1)$ & \\
\hline$\$ 39 k-\$ 47,999(2)$ & & $25.2 \%(24.1-26.3)$ & $28.9 \%(27.2-30.6)$ & \\
\hline$\$ 48 k-\$ 62,999(3)$ & & $24.4 \%(23.4-25.4)$ & $24.5 \%(22.9-26.1)$ & \\
\hline$\$ 63 k$ or more $(4)$ & & $23.2 \%(21.8-24.6)$ & $18.6 \%(16.7-20.4)$ & \\
\hline Death, in hospital & DIED & $23.4 \%(22.5-24.2)$ & $20.2 \%(18.9-21.4)$ & $<0.0001,17.32$ \\
\hline Risk of mortality & APRDRG_Risk Mortality & & & $<0.0001,64.18$ \\
\hline Minor likelihood & & $6.8 \%(6.2-7.3)$ & $10.6 \%(9.6-11.5)$ & \\
\hline Moderate likelihood & & $15.8 \%(15.1-16.5)$ & $15.4 \%(14.3-16.4)$ & \\
\hline Major likelihood & & $32.0 \%(31.1-32.9)$ & $28.8 \%(27.5-30.1)$ & \\
\hline Extreme likelihood & & $45.4 \%(44.4-46.5)$ & $45.3 \%(43.7-46.9)$ & \\
\hline Severity of illness & APRDRG_Severity & & & $<0.0001,53.30$ \\
\hline Minor loss of function & & $2.0 \%(1.7-2.2)$ & $1.7 \%(1.4-2.1)$ & \\
\hline Moderate loss of function & & $4.2 \%(3.8-4.6)$ & $5.7 \%(5.1-6.3)$ & \\
\hline Major loss of function & & $49.3 \%(48.2-50.4)$ & $43.2 \%(41.5-44.9)$ & \\
\hline Extreme loss of function & & $44.5 \%(43.5-46.6)$ & $49.3 \%(47.6-51.1)$ & \\
\hline \multicolumn{5}{|l|}{ Hospital variable } \\
\hline Control of hospital & H_CONTRL & & & $<0.0001,33.40$ \\
\hline Government, nonfederal (1) & & $13.4 \%(12.0-14.9)$ & $18.6 \%(14.7-22.5)$ & \\
\hline Private, nonprofit (2) & & $74.2 \%(72.4-76.0)$ & $75.0 \%(71.0-79.0)$ & \\
\hline Private, investor-owned (3) & & $12.3 \%(11.1-13.6)$ & $6.4 \%(5.1-7.7)$ & \\
\hline Size of hospital & HOSP_BEDSIZE & & & $<0.0001,53.43$ \\
\hline Small (1) & & $8.8 \%(7.8-9.8)$ & $4.4 \%(2.8-6.1)$ & \\
\hline Medium (2) & & $22.9 \%(21.3-24.6)$ & $14.8 \%(12.3-17.3)$ & \\
\hline Large (3) & & $68.3 \%(66.5-70.1)$ & $80.7 \%(77.7-83.7)$ & \\
\hline Location/teaching status & HOSP_LOCTEACH & & & $<0.0001,276.28$ \\
\hline Rural (1) & & $3.3 \%(3.0-3.7)$ & $1.1 \%(0.7-1.5)$ & \\
\hline Urban nonteaching (2) & & $21.5 \%(20.1-22.8)$ & $7.7 \%(6.3-9.0)$ & \\
\hline Urban teaching (3) & & $75.2 \%(73.8-76.6)$ & $91.3 \%(89.8-92.7)$ & \\
\hline
\end{tabular}

$\mathrm{HMO}=$ health maintenance organization; $\mathrm{k}=$ thousand; NA = not applicable.

* Values expressed as numbers rather than column percentages. 
TABLE 2. Transfer rates for other serious diseases in the 2013 and 2014 NIS in comparison to the transfer rates for patients with aSAH undergoing aneurysm treatment

\begin{tabular}{lrc}
\hline ICD-9-CM Code: Explanation & Total No. & Transfer In (\%) \\
\hline 39.51, 39.72, 39.75, 39.76, or 39.79: clip- \& coil-treated in aSAH cohort & 4,373 & $44.3 \%(1,938 / 4,373)$ \\
\hline 39.51: microsurgical clip ligation & 1,379 & $46.5 \%(641 / 1,379)$ \\
\hline 39.72, 39.75, 39.76, or 39.79: coil procedures & 2,994 & $43.3 \%(1,297 / 2,994)$ \\
\hline 441.6: thoracoabdominal aneurysm, ruptured & 150 & $39.3 \%$ \\
\hline 441.1: thoracic aneurysm, ruptured & 439 & $25.7 \%$ \\
\hline 441.3: abdominal aneurysm, ruptured & 2,124 & $21.4 \%$ \\
\hline 852.x: subarachnoid, subdural, \& extradural hemorrhage, following injury & 47,803 & $17.4 \%$ \\
\hline 410.x: acute myocardial infarction & 374,900 & $15.3 \%$ \\
\hline 952.x: spinal cord injury w/o evidence of spinal bone injury & 4,203 & $15.1 \%$ \\
\hline
\end{tabular}

status, location) by transfer status. Analyses utilized SAS software (version 9.4, SAS Institute Inc.).

\section{Results \\ Overall Cohort}

There was no statistically significant difference in severity scores based on transfer status among all SAH patients (Table 1). A slightly higher percentage of patients who had not been transferred died in the hospital $(23.4 \%$ nontransfers vs $20.2 \%$ transfers). This difference was not significant in the multivariate modeling.

Of the patients with aSAH requiring treatment via clip ligation or endovascular embolization, $43.3 \%$ of patients (1712/3957; data unavailable for 416 patients) were transferred from one acute care hospital to another. For context, similar transfer rates were evaluated for select acute diseases using ICD-9 codes (Table 2): myocardial infarction (ICD-9-CM 410.x); subarachnoid, subdural, and extradural hemorrhage, following injury (ICD-9-CM 852.x); spinal cord injury without evidence of spinal bone injury (ICD-9-CM 952.x); ruptured thoracic aneurysm (ICD-9CM 441.1); ruptured abdominal aneurysm (ICD-9-CM 441.3); or ruptured thoracoabdominal aneurysm (ICD9-CM 441.6). Transfer rates for neurosurgical conditions were $43 \%-46 \%$, above the comparators ranging from $15 \%-39 \%$.

Hospital location/teaching status was significantly associated with transfer status (Table 3). Patients in urban teaching hospitals were significantly more likely to have been transferred than the patients in urban nonteaching hospitals (OR 2.17, 95\% CI 1.72-2.74), but patients in rural hospitals were less likely than those in urban nonteaching hospitals to have been transferred (OR 0.8, 95\% CI $0.29-2.21)$. Hospital bed size was associated with transfer status, significantly so for large hospitals (OR 1.88, 95\% CI 1.41-2.51) but not significantly so for medium hospitals (OR 1.15, 95\% CI 0.83-1.59). Government hospitals were significantly associated with transfers compared to private investor-owned hospitals (OR 1.98, 95\% CI 1.52-2.59), as were private nonprofit hospitals (OR 1.40, 95\% CI 1.111.78).

Race was significantly associated with transfer status. Compared to whites, minorities were less likely to have been transferred (black: OR 0.80, 95\% CI 0.67-0.96; Hispanic: OR $0.58,95 \%$ CI $0.47-0.72$ ). Compared to patients in zip codes with the lowest income quartile, patients in the highest income quartile were less likely to have been transferred (OR 0.77 , 95\% CI 0.64-0.94). Primary payer, clip versus coil procedure, female sex, and age were not significantly related to transfer in the multivariate model.

\section{Microsurgical Repair}

Among patients who underwent microsurgical repair, those in urban teaching hospitals had significantly more transfers than those in urban nonteaching hospitals (OR $2.39,95 \%$ CI 1.52-3.75), whereas those in rural hospitals had fewer transfers than those in urban nonteaching hospitals, though not significantly so (OR $0.86,95 \%$ CI 0.15-4.80; Table 4). Moreover, patients who underwent microsurgery for aneurysm repair in a government hospital had higher odds of being transferred than those in private investor-owned hospitals (OR 2.24, 95\% CI 1.313.83). The effect of hospital bed size diminished in the microsurgical group. The overall $\mathrm{p}$ value was 0.0075 , but the $95 \%$ confidence interval for the odds ratios crossed 1. Race, primary payer, age, income quartile, and female sex were all nonsignificant for this group.

\section{Endovascular Embolization}

For the patients who underwent endovascular repair, those in large hospitals had greater odds of being a transfer than those in small hospitals (OR 2.17, 95\% CI 1.533.10 ), as did those in urban teaching hospitals relative to patients in urban nonteaching hospitals (OR 2.09, 95\% CI 1.59-2.75; Table 5). The 95\% confidence intervals crossed 1 for the following comparisons: medium versus small hospitals and rural versus urban nonteaching hospitals. As in the full cohort, black (OR 0.78, 95\% CI 0.63-0.97) and Hispanic (OR 0.57, 95\% CI 0.44-0.73) patients who underwent endovascular embolization were less likely to be transfers than white patients. Moreover, the patients in government-owned hospitals (OR 1.90, 95\% CI 1.39-2.60) were more likely to be transfers than the patients in private investor-owned hospitals. The patients in private nonprofit hospitals were also more likely to be transfers than the patients in private investor-owned hospitals (OR 1.38, 95\% CI 1.05-1.80). The relationship between income and transfer status mimicked that seen in the full cohort, with a significant difference in the odds of transfer only when 
TABLE 3. Multivariate model evaluating the odds of being transferred in among 3957 aSAH patients treated with clipping or coiling

\begin{tabular}{|c|c|c|c|}
\hline Variable & OR $(95 \% \mathrm{Cl})$ & $p$ Value & F-Statistic \\
\hline \multicolumn{4}{|l|}{ Hospital variable } \\
\hline $\begin{array}{l}\text { Hospital location/teaching } \\
\text { status }\end{array}$ & & $<0.0001$ & 22.9 \\
\hline $\begin{array}{l}\text { Urban teaching vs } \\
\text { urban nonteaching }\end{array}$ & $2.17(1.72-2.74)$ & & \\
\hline $\begin{array}{l}\text { Rural vs urban } \\
\text { nonteaching }\end{array}$ & $0.80(0.29-2.21)$ & & \\
\hline Hospital bed size & & $<0.0001$ & 21.0 \\
\hline Medium vs small & $1.15(0.83-1.59)$ & & \\
\hline Large vs small & $1.88(1.41-2.51)$ & & \\
\hline Hospital control & & $<0.0001$ & 14.4 \\
\hline $\begin{array}{l}\text { Government vs private } \\
\text { investor-owned }\end{array}$ & $1.98(1.52-2.59)$ & & \\
\hline $\begin{array}{l}\text { Private nonprofit vs pri- } \\
\text { vate investor-owned }\end{array}$ & $1.40(1.11-1.78)$ & & \\
\hline \multicolumn{4}{|l|}{ Patient variable } \\
\hline Race & & $<0.0001$ & 9.0 \\
\hline Black vs white & $0.80(0.67-0.96)$ & & \\
\hline Hispanic vs white & $0.58(0.47-0.72)$ & & \\
\hline Other vs white & $0.90(0.72-1.11)$ & & \\
\hline Zip code income quartile & & 0.0131 & 3.6 \\
\hline $\begin{array}{c}\$ 40 \mathrm{k}-\$ 50,999 \text { vs } \\
\$ 1-\$ 39,999\end{array}$ & $1.06(0.89-1.26)$ & & \\
\hline $\begin{array}{c}\$ 51 \mathrm{k}-\$ 65,999 \text { vs } \\
\$ 1-\$ 39,999\end{array}$ & $0.97(0.81-1.16)$ & & \\
\hline$\$ 66 k+$ vs $\$ 1-\$ 39,999$ & $0.77(0.64-0.94)$ & & \\
\hline Primary payer & & 0.18 & 1.6 \\
\hline Medicaid vs Medicare & $0.91(0.72-1.15)$ & & \\
\hline Private vs Medicare & $0.86(0.71-1.03)$ & & \\
\hline Other vs Medicare & $1.03(0.81-1.31)$ & & \\
\hline Procedure type: clip vs coil & $1.11(0.97-1.27)$ & 0.14 & 2.2 \\
\hline Female sex & $1.04(0.90-1.19)$ & 0.62 & 0.3 \\
\hline Age (per decade) & $0.99(0.93-1.05)$ & 0.69 & 0.2 \\
\hline
\end{tabular}

Reference group appears on the right in all comparisons. Data unavailable for 416 patients; therefore, data in the table reflect those obtained in $3957 \mathrm{aSAH}$ patients treated with coiling or clipping. Number of events (transfer in) was 1712/3957 (43.3\%). The C-index was 0.63 .

comparing the top and bottom quartiles (OR 0.65, 95\% CI $0.51-0.82)$, where again the patients from higher-income zip codes were less likely to be transferred.

\section{Sensitivity Analysis}

Hospital size, teaching status, and hospital type were significantly associated with transfer status in the full cohort and the two treatment subgroups. Because patients are transferred from small hospitals to large hospitals, we ran sensitivity analyses for interactions between hospital location/teaching status and hospital control by combining them into a 7-level variable. No significant interactions were noted.
TABLE 4. Multivariate model evaluating the odds of being transferred in among 1255 aSAH patients who underwent microsurgical repair of an intracranial aneurysm

\begin{tabular}{|c|c|c|c|}
\hline Variable & OR $(95 \% \mathrm{Cl})$ & $p$ Value & F-Statistic \\
\hline \multicolumn{4}{|l|}{ Hospital variable } \\
\hline $\begin{array}{l}\text { Hospital location/teaching } \\
\text { status }\end{array}$ & & 0.0005 & 7.7 \\
\hline $\begin{array}{l}\text { Urban nonteaching } \\
\text { vs urban teaching }\end{array}$ & $2.39(1.52-3.75)$ & & \\
\hline $\begin{array}{l}\text { Rural vs urban } \\
\text { teaching }\end{array}$ & $0.86(0.15-4.80)$ & & \\
\hline Hospital control & & 0.0027 & 5.9 \\
\hline $\begin{array}{l}\text { Government vs private } \\
\text { investor-owned }\end{array}$ & $2.24(1.31-3.83)$ & & \\
\hline $\begin{array}{l}\text { Private nonprofit vs pri- } \\
\text { vate investor-owned }\end{array}$ & $1.50(0.92-2.46)$ & & \\
\hline Hospital bed size & & 0.0075 & 4.9 \\
\hline Medium vs small & $0.91(0.51-1.61)$ & & \\
\hline Large vs small & $1.47(0.88-2.44)$ & & \\
\hline \multicolumn{4}{|l|}{ Patient variable } \\
\hline Race & & 0.06 & 2.5 \\
\hline Black vs white & $0.81(0.59-1.11)$ & & \\
\hline Hispanic vs white & $0.61(0.42-0.88)$ & & \\
\hline Other vs white & $0.85(0.57-1.27)$ & & \\
\hline Primary payer & & 0.14 & 1.8 \\
\hline Medicaid vs Medicare & $0.67(0.44-1.01)$ & & \\
\hline Private vs Medicare & $0.72(0.51-1.02)$ & & \\
\hline Other vs Medicare & $0.89(0.59-1.36)$ & & \\
\hline Age (per decade) & $0.90(0.81-1.00)$ & 0.0451 & 4.0 \\
\hline Zip code income quartile & & 0.67 & 0.5 \\
\hline $\begin{array}{c}\$ 40 \mathrm{k}-\$ 50,999 \text { vs } \\
\$ 1-\$ 39,999\end{array}$ & $1.20(0.88-1.62)$ & & \\
\hline $\begin{array}{c}\$ 51 \mathrm{k}-\$ 65,999 \mathrm{vs} \\
\$ 1-\$ 39,999\end{array}$ & $1.15(0.83-1.58)$ & & \\
\hline$\$ 66 k+$ vs $\$ 1-\$ 39,999$ & $1.17(0.82-1.68)$ & & \\
\hline Female sex & $1.02(0.79-1.32)$ & 0.87 & 0.03 \\
\hline
\end{tabular}

Reference group appears on the right in all comparisons. Data unavailable for 124 patients; therefore, data in the table reflect those obtained in $1255 \mathrm{aSAH}$ patients treated with microsurgical repair. Number of events (transfer in) was 575/1255 (45.8\%). The C-index was 0.63.

\section{Discussion}

These results are the first to characterize interhospital transfer for aSAH patients using a nationally representative sample. Patients with aSAH require highly specialized surgical and critical care, so evidence of a strong correlation between SAH volume and outcome supports transfer to high-volume quaternary care centers. Conversely, given the risk of early rebleeding, the transfer of patients with aSAH carries unique risks. Patients with aSAH requiring treatment are transferred at higher rates than patients with many other serious medical conditions-twice the rate of patients with acute myocardial infarction, traumatic intracranial hemorrhage, or spinal cord injury. Due to the lower 
TABLE 5. Multivariate model evaluating the odds of being transferred in among 2702 patients who underwent endovascular repair of an intracranial aneurysm

\begin{tabular}{|c|c|c|c|}
\hline Variable & OR $(95 \% \mathrm{Cl})$ & $p$ Value & F-Statistic \\
\hline \multicolumn{4}{|l|}{ Hospital variable } \\
\hline Hospital bed size & & $<0.0001$ & 17.0 \\
\hline Medium vs small & $1.33(0.89-1.98)$ & & \\
\hline Large vs small & $2.17(1.53-3.10)$ & & \\
\hline $\begin{array}{l}\text { Hospital location/teaching } \\
\text { status }\end{array}$ & & $<0.0001$ & 14.9 \\
\hline $\begin{array}{l}\text { Urban teaching vs ur- } \\
\text { ban nonteaching }\end{array}$ & $2.09(1.59-2.75)$ & & \\
\hline $\begin{array}{l}\text { Rural vs urban non- } \\
\text { teaching }\end{array}$ & $0.79(0.21-2.98)$ & & \\
\hline Race & & 0.0002 & 6.7 \\
\hline Black vs white & $0.78(0.63-0.97)$ & & \\
\hline Hispanic vs white & $0.57(0.44-0.73)$ & & \\
\hline Other vs white & $0.91(0.70-1.18)$ & & \\
\hline Hospital control & & 0.0002 & 8.6 \\
\hline $\begin{array}{l}\text { Government vs private } \\
\text { investor-owned }\end{array}$ & $1.90(1.39-2.60)$ & & \\
\hline $\begin{array}{l}\text { Private nonprofit vs pri- } \\
\text { vate investor-owned }\end{array}$ & $1.38(1.05-1.80)$ & & \\
\hline \multicolumn{4}{|l|}{ Patient variable } \\
\hline Zip code income quartile & & 0.0010 & 5.5 \\
\hline $\begin{array}{c}\$ 40 \mathrm{k}-\$ 50,999 \text { vs } \\
\$ 1-\$ 39,999\end{array}$ & $1.00(0.81-1.24)$ & & \\
\hline $\begin{array}{c}\$ 51 \mathrm{k}-\$ 65,999 \text { vs } \\
\$ 1-\$ 39,999\end{array}$ & $0.90(0.72-1.12)$ & & \\
\hline$\$ 66 k+v s \$ 1-\$ 39,999$ & $0.65(0.51-0.82)$ & & \\
\hline Primary payer & & 0.43 & 0.9 \\
\hline Medicaid vs Medicare & $1.06(0.80-1.41)$ & & \\
\hline Private vs Medicare & $0.93(0.74-1.17)$ & & \\
\hline Other vs Medicare & $1.12(0.84-1.50)$ & & \\
\hline Age (per decade) & $1.03(0.96-1.11)$ & 0.36 & 0.8 \\
\hline Female sex & $1.04(0.88-1.23)$ & 0.65 & 0.2 \\
\hline
\end{tabular}

Reference group appears on the right in all comparisons. Data unavailable for 292 patients; therefore, data in the table reflect those obtained in $2702 \mathrm{aSAH}$ patients treated with endovascular repair. Number of events (transfer in) was $1137 / 2702(42.1 \%)$. The C-index was 0.63 .

prevalence of aSAH relative to those conditions, there are likely fewer centers with the expertise necessary to treat these dangerous lesions.

There was a significant association between a patient's transfer odds and hospital teaching status. The odds of being a transfer-in patient at an urban teaching hospital were higher in the full cohort as well as each treatment subgroup. In our multivariate model, this factor held the strongest association with transfer status for both the entire cohort and the microsurgery group. In the endovascular group, hospital bed size was the strongest association with transfer status, whereas location/teaching status was the next most significant association.

Transferred patients were more likely to be transferred to a government hospital than to a private one. We found that $89 \%$ of government-owned hospitals are urban teaching hospitals. Only $17 \%$ of urban teaching hospitals are government owned and $76 \%$ are private nonprofit hospitals. These percentages support our findings in this study. Our sensitivity analysis did not show an apparent interaction between hospital location/teaching status and hospital control. Most patients with aSAH who are transferred end up in urban teaching hospitals. The least likely destination for a transfer patient-when looking at the hospital control variable-is a private investor-owned hospital.

Hospital bed size demonstrated the expected finding that patients with aSAH requiring treatment more often transfer to larger hospitals than to smaller hospitals. White patients -in the full cohort and within the two treatment subgroups-are uniformly more likely to be transferred than other races. Studies of other conditions have found similar trends, with the overall picture being one in which whites appear to have more access to the benefits of the healthcare system than minorities, even when controlling for insurance and socioeconomic status. Examples include a higher likelihood that whites will undergo surgical procedures at high-volume hospitals where a well-documented positive relationship exists between case volume and good outcome..$^{10,21}$ On the other end of an acute hospitalization are whites with moderate to severe traumatic brain injury, who are more likely to be discharged to a rehabilitation facility than are racial minorities. ${ }^{5}$ Gurwitz et al. evaluated hospital transfer rates for patients with acute myocardial infarction and found that African Americans were less likely to be transferred between acute care hospitals than whites..$^{14}$ Referral patterns may also play a role in this situation. There is evidence that different racial groups receive primary care from distinct providers. ${ }^{13}$

Patients living in zip codes with the highest median incomes are less likely to be transferred. In the current model, there was no significant interaction between race and income. Neither was there a relationship between primary payer and transfer status. This income finding could imply that patients in zip codes with the highest incomes have greater access to centers that specialize in the treatment of $\mathrm{SAH}$, compared only to patients in the lowest quartile. The difference between the lower three quartiles was not significant. Given that urban teaching hospitals are the most likely location for transfer, it is also possible that patients in highly affluent areas preferred to stay in their local hospital if treatment was available there.

Several studies have demonstrated a positive relationship between higher hospital case volumes and improved outcomes for patients with intracranial aneurysms., ${ }^{3,411,22} \mathrm{~A}$ single study reported improved outcomes at teaching hospitals. ${ }^{17}$ Other studies have failed to identify a relationship between higher hospital volume and reduced mortality. ${ }^{20,24}$ An additional work has revealed considerable variation in outcomes among high-volume centers. ${ }^{27}$

Our study has the usual limitations that apply to a retrospective administrative data set. The NIS is only as accurate as the coding performed at the contributing hospitals. A large percentage of patients (70\%) in our initial inspection had a diagnosis of SAH but no procedure to fix an aneurysm. This percentage is in line with those in other 
studies that have used the NIS ${ }^{17,22}$ to study aSAH. Many of these "nontreated" cases likely represent perimesencephalic SAH or patients who were not candidates for treatment due to a poor neurological status on presentation. Coding inaccuracies would also add to this number.

Future studies should attempt to better understand the effect of transfer characteristics on SAH outcome and should further explore racial and socioeconomic differences in transfer patterns. The recent positive trials on endovascular intervention for ischemic stroke have intensified the development of "comprehensive" stroke centers. Such centers must also demonstrate competence in dealing with intracranial aneurysms. The transport of critically ill patients is not a trivial matter, and comprehensive centers should strive for outcomes that are good enough to offset the risks of transfer. Expansion of telemedicine services is another avenue that many hospital systems are using for diseases requiring highly specialized care. These advances in technology could assist physicians on both ends in their assessment of the safety and utility of transfer for patients with aSAH.

\section{Conclusions}

Patients with aSAH requiring aneurysm treatment are transferred between acute care hospitals at a higher rate than for other dangerous conditions. They are more likely to end up in urban teaching hospitals. We did not identify a difference in transfer patterns between patients who underwent microsurgical clip ligation and those who underwent endovascular embolization. Primary payer, sex, or age did not affect transfer patterns. Income and race do play a role in the likelihood of transfer: Whites are more likely to be transferred than other races, and patients in the highest income quartile are less likely to be transferred than those in the lowest quartile.

\section{Acknowledgments}

We thank Adit Ginde, MD, for his help in the initial conception of this study. We also acknowledge the Surgical Outcomes and Applied Research program, University of Colorado School of Medicine, Aurora, CO, for supporting this project via the statistical analysis conducted by Patrick Hosokawa, MS, per the request of Christopher Roark, MD.

\section{References}

1. Addo J, Ayerbe L, Mohan KM, Crichton S, Sheldenkar A, Chen R, et al: Socioeconomic status and stroke: an updated review. Stroke 43:1186-1191, 2012

2. Ahmed I, Majeed A: Risk management during inter-hospital transfer of critically ill patients: making the journey safe. Emerg Med J 25:502-505, 2008

3. Bardach NS, Zhao S, Gress DR, Lawton MT, Johnston SC: Association between subarachnoid hemorrhage outcomes and number of cases treated at California hospitals. Stroke 33:1851-1856, 2002

4. Berman MF, Solomon RA, Mayer SA, Johnston SC, Yung PP: Impact of hospital-related factors on outcome after treatment of cerebral aneurysms. Stroke 34:2200-2207, 2003

5. Bowman SM, Martin DP, Sharar SR, Zimmerman FJ: Racial disparities in outcomes of persons with moderate to severe traumatic brain injury. Med Care 45:686-690, 2007

6. Bravata DM, Wells CK, Gulanski B, Kernan WN, Brass LM,
Long J, et al: Racial disparities in stroke risk factors: the impact of socioeconomic status. Stroke 36:1507-1511, 2005

7. Brinjikji W, Rabinstein AA, Lanzino G, Cloft HJ: Racial and ethnic disparities in the treatment of unruptured intracranial aneurysms: a study of the Nationwide Inpatient Sample 2001-2009. Stroke 43:3200-3206, 2012

8. Brinjikji W, Rabinstein AA, Nasr DM, Lanzino G, Kallmes DF, Cloft HJ: Better outcomes with treatment by coiling relative to clipping of unruptured intracranial aneurysms in the United States, 2001-2008. AJNR Am J Neuroradiol 32:1071-1075, 2011

9. Cox AM, McKevitt C, Rudd AG, Wolfe CD: Socioeconomic status and stroke. Lancet Neurol 5:181-188, 2006

10. Curry WT Jr, Carter BS, Barker FG II: Racial, ethnic, and socioeconomic disparities in patient outcomes after craniotomy for tumor in adult patients in the United States, 19882004. Neurosurgery 66:427-438, 2010

11. Dudley RA, Johansen KL, Brand R, Rennie DJ, Milstein A: Selective referral to high-volume hospitals: estimating potentially avoidable deaths. JAMA 283:1159-1166, 2000

12. Fanara B, Manzon C, Barbot O, Desmettre T, Capellier G: Recommendations for the intra-hospital transport of critically ill patients. Crit Care 14:R87, 2010

13. Fernandez A, Goldstein L: Primary care physicians who treat blacks and whites. N Engl J Med 351:2126-2127, 2004 (Letter)

14. Gurwitz JH, Goldberg RJ, Malmgren JA, Barron HV, Tiefenbrunn AJ, Frederick PDF, et al: Hospital transfer of patients with acute myocardial infarction: the effects of age, race, and insurance type. Am J Med 112:528-534, 2002

15. Isaacs SL, Schroeder SA: Class-the ignored determinant of the nation's health. N Engl J Med 351:1137-1142, 2004

16. Kissela B, Schneider A, Kleindorfer D, Khoury J, Miller R, Alwell K, et al: Stroke in a biracial population: the excess burden of stroke among blacks. Stroke 35:426-431, 2004

17. Lai PMR, Lin N, Du R: Effect of teaching hospital status on outcome of aneurysm treatment. World Neurosurg 82:380385, 385.e1-385.e6, 2014

18. Leake CB, Brinjikji W, Kallmes DF, Cloft HJ: Increasing treatment of ruptured cerebral aneurysms at high-volume centers in the United States. J Neurosurg 115:1179-1183, 2011

19. Ligtenberg JJ, Arnold LG, Stienstra Y, van der Werf TS, Meertens JH, Tulleken JE, et al: Quality of interhospital transport of critically ill patients: a prospective audit. Crit Care 9:R446-R451, 2005

20. Lindekleiv H, Mathiesen EB, Førde OH, Wilsgaard T, Ingebrigtsen T: Hospital volume and 1-year mortality after treatment of intracranial aneurysms: a study based on patient registries in Scandinavia. J Neurosurg 123:631-637, 2015

21. Liu JH, Zingmond DS, McGory ML, SooHoo NF, Ettner SL, Brook RH, et al: Disparities in the utilization of high-volume hospitals for complex surgery. JAMA 296:1973-1980, 2006

22. Pandey AS, Gemmete JJ, Wilson TJ, Chaudhary N, Thompson BG, Morgenstern LB, et al: High subarachnoid hemorrhage patient volume associated with lower mortality and better outcomes. Neurosurgery 77:462-470, 2015

23. Schievink WI, Riedinger M, Jhutty TK, Simon P: Racial disparities in subarachnoid hemorrhage mortality: Los Angeles County, California, 1985-1998. Neuroepidemiology 23:299-305, 2004

24. Seule MA, Stienen MN, Gautschi OP, Richter H, Desbiolles L, Leschka S, et al: Surgical treatment of unruptured intracranial aneurysms in a low-volume hospital-outcome and review of literature. Clin Neurol Neurosurg 114:668-672, 2012

25. Warren J, Fromm REJ Jr, Orr RA, Rotello LC, Horst HM: Guidelines for the inter- and intrahospital transport of critically ill patients. Crit Care Med 32:256-262, 2004 
26. Williams DR, Jackson PB: Social sources of racial disparities in health. Health Aff (Millwood) 24:325-334, 2005

27. Zacharia BE, Bruce SS, Carpenter AM, Hickman ZL, Vaughan KA, Richards C, et al: Variability in outcome after elective cerebral aneurysm repair in high-volume academic medical centers. Stroke 45:1447-1452, 2014

\section{Disclosures}

Dr. Seinfeld has been a consultant for Medtronic. Dr. Kumpe receives royalties from AngioDynamics Inc.

\section{Author Contributions}

Conception and design: Roark, Gritz, Hosokawa, Seinfeld, Libby. Acquisition of data: Roark, Hosokawa. Analysis and interpretation of data: Roark, Hosokawa, Seinfeld, Libby. Drafting the article: Roark, Hosokawa. Critically revising the article: all authors. Reviewed submitted version of manuscript: all authors. Approved the final version of the manuscript on behalf of all authors: Roark. Statistical analysis: Hosokawa. Administrative/technical/material support: Roark, Hosokawa, Libby. Study supervision: Roark, Libby.

\section{Correspondence}

Christopher Roark: University of Colorado, Aurora, CO. christopher.roark@ucdenver.edu. 